\title{
Bayesian Inference And Life Testing Plans For Generalized Exponential Distribution
}

\author{
Debasis Kundu * \& Biswabrata Pradhan ${ }^{\dagger}$
}

\begin{abstract}
Recently generalized exponential distribution has received considerable attentions. In this paper, we deal with the Bayesian inference of the unknown parameters of the progressively censored generalized exponential distribution. It is assumed that the scale and the shape parameters have independent gamma priors. The Bayes estimates of the unknown parameters can not be obtained in closed form. Lindley's approximation and importance sampling technique have been suggested to compute the approximate Bayes estimates. Markov Chain Monte Carlo method has been used to compute the approximate Bayes estimates and also to construct the highest posterior density credible intervals. We also provide different criteria to compare two different sampling schemes and hence to find the optimal sampling schemes. It is observed that finding the optimum censoring procedure is a computationally expensive process, and we have recommended to use the sub-optimal censoring procedure, which can be obtained very easily. Monte Carlo simulations are performed to compare the performances of the different methods and one data analysis has been performed for illustrative purposes.
\end{abstract}

Keywords: Shape parameter; Scale parameter; Markov Chain Monte Carlo; Importance Sampling; Fisher information matrix.

${ }^{*}$ Department of Mathematics and Statistics, Indian Institute of Technology Kanpur, Pin 208016, India. Corresponding author, e-mail: kundu@iitk.ac.in

${ }^{\dagger}$ SQC \& OR Unit, Indian Statistical Institute, 203 B.T. Road, Kolkata, Pin 700108, India 


\section{Introduction}

Two-parameter generalized exponential distribution (GE) was originally introduced by Gupta and Kundu [6] as a skewed distribution, and as an alternative to Weibull, gamma or lognormal distribution. Because of the shape and scale parameters, it is observed that GE distribution can take different shapes and it can be used quite effectively to analyze skewed data. Extensive work has been done by several authors on GE distribution, see for example the review article by Gupta and Kundu [8] and the references cited there.

Although extensive work has been done on the statistical inferences of the unknown parameters of the GE distribution for complete and censored sample data in the frequentest context, see for example Madi and Raqab [12], Kundu and Pradhan [10], Mitra and Kundu [13], but not much work has been done for censored sample data in the Bayesian set up. Among the different censoring schemes Type-I and Type-II are the two most popular censoring schemes, in the last few years, progressive censoring scheme has received considerable attention, see for example the book by Balakrishnan and Aggrawala [2] and also the recent excellent review article by Balakrishnan [1].

Recently in the frequentest set up, Pradhan and Kundu [15] considered the statistical inference of the unknown parameters of the generalized exponential distribution when the data are progressively censored and also proposed some optimum censoring schemes. The aim of this paper is two fold. We consider the same problem as in Pradhan and Kundu [15], but mainly from the Bayesian perspective. First we consider, the Bayes estimates of the unknown parameters under the assumptions that both the shape and the scale parameters have gamma priors and they are independently distributed. It is assumed throughout that the loss function is squared errors, although any other loss function can be easily incorporated. As expected the Bayes estimates of the unknown parameters can not be obtained 
in explicit forms. We suggest Lindley's approximations to compute the Bayes estimates of the unknown parameters, and also importance sampling technique, to construct the highest posterior density (HPD) credible intervals of the unknown parameters. We perform some simulation study to see the performances of the proposed method and also to compare with the maximum likelihood estimators. It is observed that if we have proper prior information, then the Bayesian inference has a clear advantage over the classical inference.

The second aim of this paper is to provide the methodology to compare two different sampling schemes and hence in turn, to compute the optimal sampling scheme, for a given prior distribution. Recently, Zhang and Meeker [17] and Kundu [9] discussed Bayesian life testing plan for Weibull distribution. In this paper we provide the optimal sampling plan for $G E$ distribution. It is observed that finding the optimal sampling plan is a discrete optimization problem and it is computationally quite expensive. Due to this fact we have suggested a sub-optimal plan, which can be obtained very easily.

The rest of the paper is organized as follows. In section 2, we provide the model assumption and prior distributions. Approximate Bayes estimates and the construction of HPD credible intervals are provided in section 3. Numerical results and data analysis are presented in section 4. The construction of optimal censoring scheme is presented in section 5 and finally we conclude the paper in section 6 .

\section{Problem Formulation and Prior Assumption}

It is assumed that $n$-identical units are put on a test and the lifetimes of the $n$-items are $T_{1}, \ldots, T_{n}$. It is further assumed that $T_{i}$ 's are independent and identically distributed $G E$ random variables with the $\mathrm{PDF}$

$$
f(t ; \alpha, \lambda)=\alpha \lambda\left(1-e^{-t \lambda}\right)^{\alpha-1} e^{-t \lambda} ; \quad t>0
$$


Here $\alpha>0$ and $\lambda>0$ are the shape and scale parameters respectively. The $G E$ distribution with the $\operatorname{PDF}(1)$ will be denoted by $G E(\alpha, \lambda)$. The integer $m<n$ is pre-fixed and also $R_{1}, \cdots, R_{m}$ are $m$-prefixed non-negative integers such that $R_{1}+\cdots+R_{m}+m=n$. At the time of the first failure $t_{1}, R_{1}$ of the remaining units are randomly removed. Similarly, at the time of the second failure $t_{2}, R_{2}$ of the remaining units are removed and so on. Finally, at the time of the $m$-th failure the rest of the $R_{m}=n-R_{1}-\cdots-R_{m-1}-m$ units are removed. Note that the usual Type-II censored scheme can be obtained as a special case of the progressive censoring scheme, simply by taking $R_{1}=\cdots=R_{m-1}=0$.

Now we provide the prior information on the unknown parameters. We use the following notation. A random variable $X$ is said to have a gamma distribution with the shape parameter $\beta>0$ and the scale parameter $\theta>0$, if $X$ has the following probability density function;

$$
f_{G A}(x ; \beta, \theta)=\frac{\theta^{\beta}}{\Gamma(\beta)} x^{\beta-1} e^{-\theta x} ; \quad x>0,
$$

and it will be denoted as $X \sim \operatorname{gamma}(\beta, \theta)$.

It is assumed that $\alpha$ and $\lambda$ have the following independent gamma prior distributions;

$$
\begin{array}{ll}
\pi_{1}(\alpha \mid a, b)=f_{G A}(\alpha ; b, a) ; & \alpha>0, \\
\pi_{2}(\lambda \mid c, d)=f_{G A}(\lambda ; d, c) ; & \lambda>0 .
\end{array}
$$

Here all the hyper parameters $a, b, c, d$ are known and non-negative. It may be noted that for known $\lambda,(2)$ is the conjugate prior on $\alpha$. But if both are unknown, the joint conjugate priors do not exist. Note that it is not unreasonable to assume independent gamma priors on the shape and scale parameters for a two-parameter lifetime distributions. It is due to the fact that gamma distributions are very flexible, and the Jeffrey's (non-informative) prior is a special case of this. Independent gamma priors have been used in the Bayesian analysis of Weibull distribution, see for example Berger and Sun [5] or Kundu [9]. For Bayesian 
analysis of the gamma distribution, Son and Oh [16] assumed the gamma prior on the scale parameter and independent non-informative prior on the shape parameter, which is a special case of the gamma distribution. Now based on the above priors, in the next section we obtain approximate Bayes estimates and the corresponding HPD credible intervals.

\section{Bayes Estimates and Credible Intervals}

In this section we consider the Bayes estimation of the unknown parameters, under the prior assumptions provided in (2) and (3). For computing the Bayes estimates, we have mainly assumed the squared error loss function, although any other loss function also can be incorporated. The likelihood function of the observed sample $\left\{\left(t_{1}, R_{1}\right), \ldots,\left(t_{m}, R_{m}\right)\right\}$ is

$$
l(\text { data } \mid \alpha, \lambda) \propto \alpha^{m} \lambda^{m} e^{-\lambda \sum_{i=1}^{m} t_{i}} \prod_{i=1}^{m}\left(1-e^{-\lambda t_{i}}\right)^{\alpha-1} \prod_{i=1}^{m}\left(1-\left(1-e^{-\lambda t_{i}}\right)^{\alpha}\right)^{R_{i}} .
$$

Using the joint prior distribution of $\alpha$ and $\lambda$, we obtain the joint distribution of the data, $\alpha$ and $\lambda$ as

$$
l(\text { data } \mid \alpha, \lambda) \pi_{1}(\alpha \mid a, b) \pi_{2}(\lambda \mid c, d)
$$

Based on (5), the joint posterior density of $\alpha$ and $\lambda$ given the data is

$$
l(\alpha, \lambda \mid \text { data })=\frac{l(\text { data } \mid \alpha, \lambda) \pi_{1}(\alpha \mid a, b) \pi_{2}(\lambda \mid c, d)}{\int_{0}^{\infty} \int_{0}^{\infty} l(\text { data } \mid \alpha, \lambda) \pi_{1}(\alpha \mid a, b) \pi_{2}(\lambda \mid c, d) d \alpha d \lambda}
$$

Therefore, the Bayes estimates of any function of $\alpha$ and $\lambda$ say $g(\alpha, \lambda)$ under the squared error loss function is

$$
\begin{aligned}
\widehat{g}_{B}(\alpha, \lambda) & =E_{\alpha, \lambda \mid \text { data }}(g(\alpha, \lambda)) \\
& =\int_{0}^{\infty} \int_{0}^{\infty} g(\alpha, \lambda) l(\alpha, \lambda \mid \text { data }) d \alpha d \lambda \\
& =\frac{\int_{0}^{\infty} \int_{0}^{\infty} g(\alpha, \lambda) l(\text { data } \mid \alpha, \lambda) \pi_{1}(\lambda \mid a, b) \pi_{2}(\alpha \mid c, d) d \alpha d \lambda}{\int_{0}^{\infty} \int_{0}^{\infty} l(\text { data } \mid \alpha, \lambda) \pi_{1}(\lambda \mid a, b) \pi_{2}(\alpha \mid c, d) d \alpha d \lambda} .
\end{aligned}
$$


It is not possible to compute (7) analytically in this case. Two approaches are suggested here to approximate (7), namely (a) Lindley's approximation and (b) Importance Sampling Procedure.

\subsection{LindLEY'S APPROXimATion}

Lindley proposed his procedure to approximate the ratio of two integrals such as (7). This has been used by several authors. Based on Lindley's approximation, the Bayes estimates of $\alpha$ and $\lambda$ under the squared error loss function are

$$
\begin{aligned}
\widehat{\alpha}_{B}= & \widehat{\alpha}+\frac{1}{2}\left(l_{30} \tau_{11}^{2}+l_{03} \tau_{21} \tau_{22}+3 l_{21} \tau_{11} \tau_{12}+l_{12}\left(\tau_{22} \tau_{11}+2 \tau_{21}^{2}\right)\right) \\
& +\left(\frac{b-1}{\widehat{\alpha}}-a\right) \tau_{11}+\left(\frac{d-1}{\widehat{\lambda}}-c\right) \tau_{12}, \\
\widehat{\lambda}_{B}= & \widehat{\lambda}+\frac{1}{2}\left(l_{30} \tau_{12} \tau_{11}+l_{03} \tau_{22}^{2}+l_{21}\left(\tau_{11} \tau_{22}+2 \tau_{12}^{2}\right)+3 l_{12} \tau_{22} \tau_{21}\right) \\
& +\left(\frac{b-1}{\widehat{\alpha}}-a\right) \tau_{21}+\left(\frac{d-1}{\widehat{\lambda}}-c\right) \tau_{22} .
\end{aligned}
$$

Here $\widehat{\alpha}$ and $\hat{\lambda}$ are the MLEs of $\alpha$ and $\lambda$ respectively, and $a, b, c, d$ are the known hyperparameters. The explicit expressions of $\tau_{11}, \tau_{12}, \tau_{21}, \tau_{22}, \tau_{30}, \tau_{03}, l_{21}, l_{12}$ are provided in the Appendix A.

\subsection{Importance SAMPLing}

In the previous subsection we obtain the Bayes estimates of the unknown parameters using the Lindley's approximation method. Unfortunately, using Lindley's method it is not possible to compute the highest posterior density (HPD) credible intervals. In this subsection, we

propose importance sampling procedure to compute the Bayes estimates and also to construct the HPD credible intervals. 
Under the assumptions on the priors of $\alpha$ and $\lambda$ as given in (2) and (3), the posterior density function of $\alpha$ and $\lambda$ can be written as

$$
\begin{aligned}
l(\alpha, \lambda \mid \text { data }) & \propto \alpha^{b+m-1} e^{-\alpha\left(a-\sum_{i=1}^{m} \ln \left(1-e^{-\lambda t_{i}}\right)\right)} \lambda^{d+m-1} e^{-\lambda\left(c+\sum_{i=1}^{m} t_{i}\right)} \prod_{i=1}^{m} \frac{\left(1-\left(1-e^{-\lambda t_{i}}\right)^{\alpha}\right)^{R_{i}}}{\left(1-e^{-\lambda t_{i}}\right)} \\
& \propto f_{G A}\left(\alpha ; b+m, a-\sum_{i=1}^{m} \ln \left(1-e^{-\lambda t_{i}}\right)\right) f_{G A}\left(\lambda ; d+m, c+\sum_{i=1}^{m} t_{i}\right) \times h(\alpha, \lambda),(10)
\end{aligned}
$$

where

$$
h(\alpha, \lambda)=\frac{\prod_{i=1}^{m} \frac{\left(1-\left(1-e^{-\lambda t_{i}}\right)^{\alpha}\right)^{R_{i}}}{\left(1-e^{-\lambda t_{i}}\right)}}{\left(a-\sum_{i=1}^{m} \ln \left(1-e^{-\lambda t_{i}}\right)\right)^{b+m}} .
$$

Using (10), it is possible to use the importance sampling procedure to compute the Bayes estimates of any function of $\alpha$ and $\lambda$, say $g(\alpha, \lambda)$ and also to construct the corresponding HPD credible intervals.

Let us denote the right hand side of (10) as $l_{N}(\alpha, \lambda \mid$ data $)$. Therefore, $l_{N}(\alpha, \lambda \mid$ data $)$ and $l(\alpha, \lambda \mid$ data $)$ differ only by the proportionality constant. The, Bayes estimate of $g(\alpha, \lambda)$ under the squared error loss function is

$$
\widehat{g}_{B}(\alpha, \lambda)=\frac{\int_{0}^{\infty} \int_{0}^{\infty} g(\alpha, \lambda) l_{N}(\alpha, \lambda \mid d a t a) d \alpha d \lambda}{\int_{0}^{\infty} \int_{0}^{\infty} l_{N}(\alpha, \lambda \mid d a t a) d \alpha d \lambda}
$$

It is clear from (12) that to approximate $\widehat{g}_{B}(\alpha, \lambda)$, using the importance sampling procedure one need not compute the normalizing constant. We use the following procedure:

- Step 1: Generate

$$
\lambda_{1} \sim \operatorname{gamma}\left(d+m, c+\sum_{i=1}^{m} t_{i}\right) \quad \text { and } \quad \alpha_{1} \mid \lambda_{1} \sim \operatorname{gamma}\left(b+m, a-\sum_{i=1}^{m} \ln \left(1-e^{-\lambda_{1} t_{i}}\right)\right) .
$$

- Step 2: Repeat this procedure to obtain $\left(\alpha_{1}, \lambda_{1}\right), \ldots,\left(\alpha_{N}, \lambda_{N}\right)$.

- Step 3: The approximate value of (12) can be obtained as

$$
\frac{\sum_{i=1}^{N} g\left(\alpha_{i}, \lambda_{i}\right) h\left(\alpha_{i}, \lambda_{i}\right)}{\sum_{i=1}^{N} h\left(\alpha_{i}, \lambda_{i}\right)} .
$$


Now we would like to obtain the highest posterior density (HPD) credible intervals of $\alpha$ and $\lambda$ using the generated importance sampling procedure. We illustrate the procedure for $\alpha$ but it can be similarly implemented for $\lambda$ also.

Suppose $a_{p}$ is such that

$$
P\left[\alpha \leq a_{p} \mid \text { data }\right]=p .
$$

Now consider the following function

$$
g(\alpha, \lambda)=\left\{\begin{array}{lll}
1 & \text { if } & \alpha \leq a_{p} \\
0 & \text { if } & \alpha>a_{p}
\end{array}\right.
$$

Clearly,

$$
E(g(\alpha, \lambda) \mid \text { data })=p
$$

Therefore, an approximate Bayes estimate of $a_{p}$ under the squared error loss function can be obtained from the generated sample $\left\{\left(\alpha_{1}, \lambda_{1}\right), \ldots,\left(\alpha_{N}, \lambda_{N}\right)\right\}$ as follows. Let

$$
w_{i}=\frac{h\left(\alpha_{i}, \lambda_{i}\right)}{\sum_{i=1}^{N} h\left(\alpha_{i}, \lambda_{i}\right)} ; \quad i=1, \ldots, N
$$

Rearrange $\left\{\left(\alpha_{1}, w_{1}\right), \ldots,\left(\alpha_{N}, w_{N}\right)\right\}$ as $\left\{\left(\alpha_{(1)}, w_{(1)}\right), \ldots,\left(\alpha_{(N)}, w_{(N)}\right)\right\}$, where $\alpha_{(1)}<\ldots<$ $\alpha_{(N)}$. Note that $w_{(i)}$ 's are not ordered, they are just associated with $\alpha_{(i)}$. Then an Bayes estimate of $a_{p}$ is

$$
\widehat{a}_{p}=\alpha_{\left(N_{p}\right)}
$$

where $N_{p}$ is the integer satisfying

$$
\sum_{i=1}^{N_{p}} w_{(i)} \leq p<\sum_{i=1}^{N_{p}+1} w_{(i)} .
$$

Now using the above procedure a $100(1-\gamma) \%$ credible interval of $\alpha$ can be obtained as $\left(\widehat{a}_{\delta}, \widehat{a}_{\delta+1-\gamma}\right), \quad$ for $\quad \delta=w_{(1)}, w_{(1)}+w_{(2)}, \ldots, \sum_{i=1}^{N_{1-\gamma}} w_{(i)}$. Therefore, an $100(1-\gamma) \% \operatorname{HPD}$ credible interval of $\alpha$ becomes

$$
\left(\widehat{a}_{\delta^{*}}, \quad\left(\widehat{a}_{\delta^{*}+1-\gamma}\right)\right.
$$


where $\delta^{*}$ is such that

$$
\widehat{a}_{\delta^{*}+1-\gamma}-\widehat{a}_{\delta^{*}} \leq \widehat{a}_{\delta+1-\gamma}-\widehat{a}_{\delta} \quad \text { for all } \delta
$$

\section{Numerical Experiments and Data Analysis}

\subsection{Numerical EXPERIMENTS}

In this subsection, we present some experimental results to observe the behavior of the proposed methods for different sample sizes, for different priors and for different sampling schemes. All the computations are performed at the Indian Statistical Institute Kolkata. We have considered different sample sizes; $n=20,25,30$, different effective sample sizes; $m=10$ and 15, different hyper-parameters $(a, b, c, d)$ and ten ([1]- [10]) different sampling schemes. In all cases we have used $\alpha=1.5$ and $\lambda=1.0$.

For a particular, $n, m$ and a sampling scheme, using the algorithm proposed by Balakrishnan and Sandhu [3] we have generated the progressively censored generalized exponential distribution. First we have used the non-informative gamma priors for both the shape and scale parameters, i.e, when all the hyper-parameters are zero and we call it as Prior-1. Note that as the hyper-parameters go to zero, the prior density becomes inversely proportional to its argument and also becomes improper. This density is commonly used as an improper prior for parameters in the range of zero to infinity and this prior is not specifically related to gamma priors only. It should also be mentioned that even if we take non-proper prior, the joint posterior density of $\alpha$ and $\lambda$ is still proper.

For comparison purposes, we have considered informative gamma priors also. For example we have considered Prior-2: $a=1.5, b=2.25, c=5$ and $d=5$. Purposely, we have taken the

prior means to be same as the original means. Note that Prior-1 and Prior-2 are two extreme priors. In one case it is totally non-informative (Prior-1) and in the other case, prior means 
are exactly equal to the original means. In practice most of the priors will be in between these two extremes.

In all cases squared error loss functions has been used for computing the Bayes estimates. For a particular censoring scheme, we compute MLEs and the Bayes estimates using Lindley's approximations. We compute asymptotic confidence intervals of $\alpha$ and $\lambda$ based on the Fisher information matrix. We also compute the Bayes estimates and 95\% highest posterior density (HPD) credible intervals based on 1000 importance sampling. We replicate the process for 2000 times and average estimates, mean squared error, average confidence length / credible length and $95 \%$ coverage percentages are reported in Table $1,2 \& 3$.

Note that the sampling schemes, [1], [3], [5], [7], [9] are the usual Type-II censoring schemes, i.e., $n-m$ items are removed at the time of the $m$-th failure. The sampling schemes [2], [4], [6], [8], [10] are just the opposite of the Type-II sampling schemes, i.e., $n-m$ items are removed at the time of the first failure. Let us denote this censoring as Type-III censoring scheme, as in Pradhan and Kundu [15]. For fixed $n$ and $m$, the expected experimental time of the Type-II censoring schemes are less than the corresponding Type-III censoring schemes. The expected experimental time of any other censoring scheme will be always between these two extremes, for fixed $n$ and $m$.

Some of the foregoing points are made very clear from the tables. It is clear that the MLEs and the Bayes estimates using Lindley's approximation behave very much in a similar manner particularly, in case of non-informative priors. The Bayes estimates obtained using MCMC are slightly less biased than the MLEs or the Bayes estimates obtained using Lindley's approximations, but the mean squared errors are almost the same. Interestingly, similar behavior has been observed for prior-2 also. Even in case of prior-2, the biases of the Bayes estimates obtained using MCMC are slightly less than the Bayes estimates obtained using Lindley's approximations, but their mean squared errors are almost the same. Now 
Table 1: Average values of the maximum likelihood estimators and Bayes estimators under prior-1 and the corresponding mean squared errors (in parenthesis).

\begin{tabular}{|c|c|c|c|c|c|c|c|c|c|}
\hline \multirow[t]{2}{*}{$n$} & \multirow[t]{2}{*}{$m$} & \multirow[t]{2}{*}{ Scheme } & \multirow[t]{2}{*}{ No. } & \multicolumn{2}{|c|}{ MLEs } & \multicolumn{2}{|c|}{ Bayes (MCMC) } & \multicolumn{2}{|c|}{ Bayes (Lindley) } \\
\hline & & & & $\alpha$ & $\lambda$ & $\alpha$ & $\lambda$ & $\alpha$ & $\lambda$ \\
\hline 20 & 10 & $\left(9^{*} 0,10\right)$ & {$[1]$} & $\begin{array}{r}1.3590 \\
(0.0966)\end{array}$ & $\begin{array}{l}1.1489 \\
(0.0804)\end{array}$ & $\begin{array}{r}1.5835 \\
(0.1022)\end{array}$ & $\begin{array}{l}1.1201 \\
(0.0938)\end{array}$ & $\begin{array}{r}1.3431 \\
(0.1068)\end{array}$ & $\begin{array}{l}1.1312 \\
(0.0993)\end{array}$ \\
\hline 20 & 10 & $\left(10,9^{*} 0\right)$ & {$[2]$} & $\begin{array}{r}1.3958 \\
(0.0729)\end{array}$ & $\begin{array}{l}0.8817 \\
(0.0711)\end{array}$ & $\begin{array}{r}1.5448 \\
(0.0715)\end{array}$ & $\begin{array}{l}1.0191 \\
(0.0670)\end{array}$ & $\begin{array}{r}1.3532 \\
(0.0762)\end{array}$ & $\begin{array}{l}0.8299 \\
(0.0733)\end{array}$ \\
\hline 25 & 10 & $(9 * 0,15)$ & [3] & $\begin{array}{r}1.2394 \\
(0.0835)\end{array}$ & $\begin{array}{l}0.7604 \\
(0.0513)\end{array}$ & $\begin{array}{r}1.5920 \\
(0.0904)\end{array}$ & $\begin{array}{l}1.1924 \\
(0.0477)\end{array}$ & $\begin{array}{r}1.1891 \\
(0.0888)\end{array}$ & $\begin{array}{l}0.6972 \\
(0.0502)\end{array}$ \\
\hline 25 & 10 & $(15,9 * 0)$ & {$[4]$} & $\begin{array}{r}1.2683 \\
(0.0711)\end{array}$ & $\begin{array}{l}0.7856 \\
(0.0516)\end{array}$ & $\begin{array}{r}1.4532 \\
(0.0695)\end{array}$ & $\begin{array}{l}0.9868 \\
(0.0498)\end{array}$ & $\begin{array}{r}1.2666 \\
(0.0739)\end{array}$ & $\begin{array}{l}0.7602 \\
(0.0561)\end{array}$ \\
\hline 25 & 15 & $\left(14^{*} 0,10\right)$ & {$[5]$} & $\begin{array}{r}1.4143 \\
(0.0526)\end{array}$ & $\begin{array}{l}0.9635 \\
(0.0742)\end{array}$ & $\begin{array}{r}1.5158 \\
(0.0513)\end{array}$ & $\begin{array}{l}1.0633 \\
(0.0703)\end{array}$ & $\begin{array}{r}1.4651 \\
(0.0566)\end{array}$ & $\begin{array}{l}0.9134 \\
(0.0740)\end{array}$ \\
\hline 25 & 15 & $\left(10,14^{*} 0\right)$ & [6] & $\begin{array}{r}1.4123 \\
(0.0449)\end{array}$ & $\begin{array}{l}0.8971 \\
(0.0503)\end{array}$ & $\begin{array}{r}1.4643 \\
(0.0410)\end{array}$ & $\begin{array}{l}0.9878 \\
(0.0540)\end{array}$ & $\begin{array}{r}1.4070 \\
(0.0428)\end{array}$ & $\begin{array}{l}0.8752 \\
(0.0519)\end{array}$ \\
\hline 30 & 10 & $(9 * 0,20)$ & {$[7]$} & $\begin{array}{r}1.1654 \\
(0.0775)\end{array}$ & $\begin{array}{l}0.6829 \\
(0.0461)\end{array}$ & $\begin{array}{c}1.5531 \\
(.0794)\end{array}$ & $\begin{array}{c}1.2538 \\
(0.0501)\end{array}$ & $\begin{array}{r}1.1159 \\
(0.0789)\end{array}$ & $\begin{array}{l}0.6263 \\
(0.0523)\end{array}$ \\
\hline 30 & 10 & $(20,9 * 0)$ & [8] & $\begin{array}{r}1.2150 \\
(0.0599)\end{array}$ & $\begin{array}{l}0.7339 \\
(0.0387)\end{array}$ & $\begin{array}{r}1.4629 \\
(0.0508)\end{array}$ & $\begin{array}{l}0.9947 \\
(0.0310)\end{array}$ & $\begin{array}{r}1.4163 \\
(0.0579)\end{array}$ & $\begin{array}{l}0.9122 \\
(0.0325)\end{array}$ \\
\hline 30 & 15 & $\left(14^{*} 0,15\right)$ & [9] & $\begin{array}{r}1.3043 \\
(0.0575)\end{array}$ & $\begin{array}{l}0.8099 \\
(0.0458)\end{array}$ & $\begin{array}{r}1.5624 \\
(0.0512)\end{array}$ & $\begin{array}{l}1.1518 \\
(0.0424)\end{array}$ & $\begin{array}{r}1.3070 \\
(0.0519)\end{array}$ & $\begin{array}{l}0.8025 \\
(0.0478)\end{array}$ \\
\hline 30 & 15 & $\left(15,14^{*} 0\right)$ & [10] & $\begin{array}{r}1.3260 \\
(0.0456)\end{array}$ & $\begin{array}{l}0.8321 \\
(0.0242)\end{array}$ & $\begin{array}{r}1.4883 \\
(0.0399)\end{array}$ & $\begin{array}{l}0.9853 \\
(0.0216)\end{array}$ & $\begin{array}{r}1.3245 \\
(0.0417)\end{array}$ & $\begin{array}{l}0.8135 \\
(0.0236)\end{array}$ \\
\hline
\end{tabular}

comparing the results for prior-1 and prior-2, it is clear that the Bayes estimates based on informative priors behave much better than the Bayes estimates based on non-informative priors or MLEs in terms of biases, MSEs and lengths of credible intervals. Therefore, if the prior information are available, then we should use the Bayes estimates, otherwise MLEs may be used to avoid the computational cost.

\subsection{Data Analysis}

In this subsection we provide a data analysis for illustrative purposes. The data have been taken from Lawless [11] and it represents the failure or censoring times of 36 appliances 
Table 2: Average values of the Bayes estimators under prior-2 and the corresponding mean squared errors (in parenthesis).

\begin{tabular}{|c|c|c|c|rl|rl|}
\hline$n$ & $m$ & Scheme & No. & Bayes $($ MCMC) & \multicolumn{2}{|c|}{ Bayes(Lindley) } \\
& & & & $\alpha$ & \multicolumn{1}{c|}{$\lambda$} & $\alpha$ & \multicolumn{1}{c|}{$\lambda$} \\
\hline 20 & 10 & $\left(9^{*} 0,10\right)$ & {$[1]$} & 1.5230 & 1.0055 & 1.4421 & 0.8686 \\
& & & & $(0.0943)$ & $(0.0347)$ & $(0.0993)$ & $(0.0584)$ \\
\hline 20 & 10 & $\left(10,9^{*} 0\right)$ & {$[2]$} & 1.5428 & 1.0088 & 1.4596 & 0.8875 \\
& & & & $(0.0627)$ & $(0.0400)$ & $(0.0710)$ & $(0.0429)$ \\
\hline 25 & 10 & $\left(9^{*} 0,15\right)$ & {$[3]$} & 1.4899 & 1.0512 & 1.5212 & 0.9528 \\
& & & & $(0.0752)$ & $(0.0377)$ & $(0.0720)$ & $(0.0355)$ \\
\hline 25 & 10 & $\left(15,9^{*} 0\right)$ & {$[4]$} & 1.4893 & 1.0015 & 1.4481 & 0.8777 \\
& & & & $(0.0739)$ & $(0.0356)$ & $(0.0698)$ & $(0.0338)$ \\
\hline 25 & 15 & $\left(14^{*} 0,10\right)$ & {$[5]$} & 1.4906 & 1.0044 & 1.3960 & 0.8866 \\
& & & & $(0.0415)$ & $(0.0304)$ & $(0.0437)$ & $(0.0289)$ \\
\hline 25 & 15 & $\left(10,14^{*} 0\right)$ & {$[6]$} & 1.4981 & 0.9961 & 1.4461 & 0.9031 \\
& & & & $(0.0375)$ & $(0.0239)$ & $(0.0395)$ & $(0.0201)$ \\
\hline 30 & 10 & $\left(9^{*} 0,20\right)$ & {$[7]$} & 1.4752 & 1.0871 & 1.5483 & 0.9966 \\
& & & & $(0.0767)$ & $(0.0457)$ & $(0.0706)$ & $(0.0418)$ \\
\hline 30 & 10 & $\left(20,9^{*} 0\right)$ & {$[8]$} & 1.4896 & 0.9958 & 1.4261 & 0.8615 \\
& & & & $(0.0534)$ & $(0.0384)$ & $(0.0501)$ & $(0.0319)$ \\
\hline 30 & 15 & $\left(14^{*} 0,15\right)$ & {$[9]$} & 1.4881 & 1.0507 & 1.4257 & 0.8921 \\
& & & & $(0.0478)$ & $(0.0361)$ & $(0.0439)$ & $(0.0315)$ \\
\hline 30 & 15 & $\left(15,14^{*} 0\right)$ & {$[10]$} & 1.5156 & 0.9987 & 1.4117 & 0.8812 \\
& & & & $(0.0337)$ & $(0.0163)$ & $(0.0362)$ & $(0.0210)$ \\
\hline
\end{tabular}

subjected to an automatic life tests. The data given below consists of only the failure times: 11, 35, 49, 170, 329, 381, 708, 958, 1062, 1167, 1594, 1925, 1990, 2223, 2327, 2400, 2451, 2471, 2551, 2565, 2568, 2694, 2702, 2761, 2831, 3034, 3059, 3112, 3214, 3478, 3504, 4329, 6367, 6967, 7846, 13403. The same data set is analyzed by Pradhan and Kundu [15]. It is observed in Pradhan and Kundu [15] that the GE distribution fits very well to the complete data in terms of Kolmogorov-Smirnov distance.

For illustrative purposes, we have generated three different progressively censored samples using three different sampling schemes with $n=36$ data with $m=12$, as follows: 
Table 3: Average confidence length / credible interval of the MLES / Bayes estimators and the $95 \%$ coverage percentage (in parenthesis).

\begin{tabular}{|c|c|c|c|c|c|c|c|c|c|}
\hline \multirow[t]{2}{*}{$n$} & \multirow[t]{2}{*}{$m$} & \multirow[t]{2}{*}{ Scheme } & \multirow[t]{2}{*}{ No. } & \multicolumn{2}{|c|}{ MLEs } & \multicolumn{2}{|c|}{$\begin{array}{c}\text { Bayes } \\
\text { Prior-1 }\end{array}$} & \multicolumn{2}{|c|}{$\begin{array}{c}\text { Bayes } \\
\text { Prior-2 }\end{array}$} \\
\hline & & & & $\alpha$ & $\lambda$ & $\alpha$ & $\lambda$ & $\alpha$ & $\lambda$ \\
\hline 20 & 10 & $\left(9^{*} 0,10\right)$ & [1] & $\begin{array}{r}2.1172 \\
(94)\end{array}$ & $\begin{array}{l}1.5212 \\
(93)\end{array}$ & $\begin{array}{r}1.7585 \\
(97)\end{array}$ & $\begin{array}{l}1.9554 \\
(96)\end{array}$ & $\begin{array}{r}1.5137 \\
(94)\end{array}$ & $\begin{array}{l}1.5238 \\
(96)\end{array}$ \\
\hline 20 & 10 & $(10,9 * 0)$ & {$[2]$} & $\begin{array}{r}1.9107 \\
(96)\end{array}$ & $\begin{array}{l}1.2561 \\
(92)\end{array}$ & $\begin{array}{r}1.7461 \\
(97)\end{array}$ & $\begin{array}{l}1.1059 \\
(92)\end{array}$ & $\begin{array}{r}1.6116 \\
(97)\end{array}$ & $\begin{array}{l}0.9727 \\
(92)\end{array}$ \\
\hline 25 & 10 & $\left(9^{*} 0,15\right)$ & {$[3]$} & $\begin{array}{r}1.7912 \\
(94)\end{array}$ & $\begin{array}{l}1.4805 \\
(94)\end{array}$ & $\begin{array}{r}1.4650 \\
(92)\end{array}$ & $\begin{array}{l}1.2611 \\
(96)\end{array}$ & $\begin{array}{r}1.2808 \\
(92)\end{array}$ & $\begin{array}{l}1.1828 \\
(98)\end{array}$ \\
\hline 25 & 10 & $\left(15,9^{*} 0\right)$ & {$[4]$} & $\begin{array}{r}1.6625 \\
(95)\end{array}$ & $\begin{array}{l}1.1734 \\
(91)\end{array}$ & $\begin{array}{r}1.6080 \\
(97)\end{array}$ & $\begin{array}{l}1.1102 \\
(92)\end{array}$ & $\begin{array}{r}1.4837 \\
(98)\end{array}$ & $\begin{array}{l}0.9843 \\
(92)\end{array}$ \\
\hline 25 & 15 & $\left(14^{*} 0,10\right)$ & {$[5]$} & $\begin{array}{r}1.8680 \\
(96)\end{array}$ & $\begin{array}{l}1.4404 \\
(94)\end{array}$ & $\begin{array}{r}1.4662 \\
(96)\end{array}$ & $\begin{array}{l}1.3192 \\
(96)\end{array}$ & $\begin{array}{r}1.2677 \\
(97)\end{array}$ & $\begin{array}{l}1.0904 \\
(97)\end{array}$ \\
\hline 25 & 15 & $\left(10,14^{*} 0\right)$ & [6] & $\begin{array}{r}1.7281 \\
(96) \\
\end{array}$ & $\begin{array}{l}1.0456 \\
(96)\end{array}$ & $\begin{array}{r}1.4432 \\
(97) \\
\end{array}$ & $\begin{array}{l}0.9566 \\
(92)\end{array}$ & $\begin{array}{r}1.3607 \\
(98)\end{array}$ & $\begin{array}{l}0.8563 \\
(95)\end{array}$ \\
\hline 30 & 10 & $\left(9^{*} 0,20\right)$ & {$[7]$} & $\begin{array}{r}1.6045 \\
(95)\end{array}$ & $\begin{array}{l}1.4169 \\
(93)\end{array}$ & $\begin{array}{r}1.8660 \\
(91)\end{array}$ & $\begin{array}{l}1.6873 \\
(92)\end{array}$ & $\begin{array}{r}1.3725 \\
(93)\end{array}$ & $\begin{array}{l}1.0724 \\
(93)\end{array}$ \\
\hline 30 & 10 & $(20,9 * 0)$ & [8] & $\begin{array}{r}1.5009 \\
(96)\end{array}$ & $\begin{array}{l}1.1044 \\
(92)\end{array}$ & $\begin{array}{r}1.6537 \\
(93)\end{array}$ & $\begin{array}{l}1.4036 \\
(93)\end{array}$ & $\begin{array}{r}1.9274 \\
(98)\end{array}$ & $\begin{array}{l}1.1467 \\
(99)\end{array}$ \\
\hline 30 & 15 & $\left(14^{*} 0,15\right)$ & [9] & $\begin{array}{r}1.6799 \\
(98)\end{array}$ & $\begin{array}{l}1.2398 \\
(94)\end{array}$ & $\begin{array}{r}1.7518 \\
(92)\end{array}$ & $\begin{array}{l}1.3334 \\
(92)\end{array}$ & $\begin{array}{r}1.4921 \\
(95)\end{array}$ & $\begin{array}{l}0.9770 \\
(92)\end{array}$ \\
\hline 30 & 15 & $\left(15,14^{*} 0\right)$ & {$[10]$} & $\begin{array}{r}1.5444 \\
(95)\end{array}$ & $\begin{array}{l}0.9998 \\
(94)\end{array}$ & $\begin{array}{r}1.7473 \\
(95)\end{array}$ & $\begin{array}{l}1.0205 \\
(92)\end{array}$ & $\begin{array}{r}1.7166 \\
(97)\end{array}$ & $\begin{array}{l}0.9013 \\
(97)\end{array}$ \\
\hline
\end{tabular}

Censoring Scheme 1: $(15,5,4,9 * 0)$. We obtain the following progressively censored sample: 11, 35, 49, 329, 1062, 1167, 1594, 1990, 2451, 2471, 2551, 3059.

Censoring Scheme 2: $\left(11^{*} 0,24\right)$. We obtain the following progressively censored sample: 11 , 35, 49, 170, 329, 381, 708, 958, 1062, 1167, 1594, 1925.

Censoring Scheme 3: $\left(24,11^{*} 0\right)$. We obtain the 11, 35, 49, 329, 381, 958, 1062, 1594, 1925, $2223,2451,2471$.

For each of the censoring scheme, we compute MLEs and Bayes estimates of $\alpha$ and $\lambda$. Since we do not have any prior information, we take $a=b=c=d=0$ for Bayes estimates. 
The estimate of the parameters are given in Table 4. It is clear that without the presence of

Table 4: MLEs and Bayes estimates of the parameters of generalized exponential distribution for the appliances data under different progressive censoring schemes.

\begin{tabular}{|c|cc|cc|cc|}
\hline Censoring & \multicolumn{2}{|c|}{ MLEs } & \multicolumn{2}{|c|}{ Bayes (Lindley) } & \multicolumn{2}{|c|}{ Bayes (MCMC) } \\
Scheme & $\alpha$ & $\lambda$ & $\alpha$ & $\lambda$ & $\alpha$ & $\lambda$ \\
\hline 1 & 0.89532 & 0.00074 & 0.89140 & 0.00072 & 0.88301 & 0.00066 \\
\hline 2 & 0.79080 & 0.00020 & 0.72308 & 0.00017 & 0.71086 & 0.00025 \\
\hline 3 & 0.88723 & 0.00093 & 0.87542 & 0.00091 & 0.86982 & 0.00087 \\
\hline
\end{tabular}

any prior knowledge the MLEs and Bayes estimates behave in a very similar manner in all the cases considered.

\section{Optimum Progressive Censoring Scheme}

For a practitioner, finding the best progressive censoring scheme is quite important. Recently finding the optimal progressive censoring scheme has received some attention in the statistical literature, see for example Balasooriya and Balakrishnan [4], Ng, Chan and Balakrishnan [14], Kundu [9] and the references cited there.

In most of the practical situation, the sample size $n$ is fixed, depending on the available resource. If the practitioner has a choice on the effective sample size $m$, the obvious choice should be $m=n$, to achieve the maximum efficiency in estimating the unknown parameters. If both the sample size $n$ and the effective sample size $m<n$ are fixed in advance, a natural question arises how to choose a particular censoring scheme. Should one choose just based on convenience, or based on some statistical criterion.

Before progressing further, first we need to know what are the possible sampling schemes and how do we compare two censoring schemes. If somebody has a choice on $n$ and $m$, therefore, the obvious choice will be $m=n$ and $n$ should be as large as possible. But 
in most of the practical situations, the experimenter may not have a choice on $n$ and $m$. Therefore, possible censoring schemes mean, for fixed sample size $n$ and effective sample size $m$, different choices of $\left\{R_{1}, \ldots, R_{m}\right\}$, such that

$$
R_{1}+\ldots+R_{m}=n
$$

The problem boils down as follows: for fixed $n$ and $m$ choose that particular censoring scheme which is optimum. To define an optimum censoring scheme, first we need to define a criterion, based on which the optimality can be defined. If time is the only criterion, i.e., the expected experimental time should be minimum, then clearly the usual type-II censoring scheme $\left(R_{1}=\ldots=R_{m-1}=0\right)$ is the optimum criterion. In this paper, we define the optimality in terms of the information measure. We would like to choose that particular censoring scheme which provides the maximum information of the unknown parameters. A progressive censoring scheme $R^{(1)}=\left\{R_{1}^{(1)}, \ldots, R_{m}^{(1)}\right\}$ is better than $R^{(2)}=\left\{R_{1}^{(2)}, \ldots, R_{m}^{(2)}\right\}$, if $R^{(1)}$ provides more information than $R^{(2)}$ about the unknown parameters.

Naturally the questions come how to define the information measure of the unknown parameters for a particular censoring scheme and how to compare two different censoring schemes. In these respects considering the corresponding Fisher information matrices and compare them seem to be the natural choice. If only one parameter is unknown then comparing the corresponding Fisher information measures is quite simple. It is only the comparison of two real numbers. If more than one unknown parameters are present, then one needs to compare two Fisher information matrices. Comparison of the two Fisher information matrices is not a trivial task. Some of the existing choices are to compare the traces or the determinants of two matrices. Unfortunately in presence of the shape and scale parameters, it has been observed, see Gupta and Kundu [7], that the trace or the determinant are not scale invariant. Therefore, it quite likely that a particular censoring scheme may be optimum with respect to a given unit, but if we change the unit of the data, then it may not remain 
the optimum scheme, which is not very desirable. To avoid this problem, using the ideas of Zhang and Meeker [17] and Kundu [9], in the next subsection we propose two different criteria.

\subsection{CRITERIA}

In this subsection we propose two criteria to compare two censoring schemes and that can be used to find the optimum censoring scheme. Both the criteria are based on the estimation of the precision of the $p$-th $(0<p<1)$ quantile. The $p$-th quantile of the $G E(\alpha, \lambda)$ is

$T_{p}=-\frac{1}{\lambda} \ln \left(1-p^{\frac{1}{\alpha}}\right)$. We define two different information measures for a given censoring scheme, say $R=\left(R_{1}, \ldots, R_{m}\right)$, based on estimating $\ln T_{p}$, as it was used by Zhang and Meeker [17] and Kundu [9]. From the two different information measures, we can easily define two different criteria and they can be easily used to compare two different censoring schemes also.

CRIterion 1: First let us define the first information measure for a given sampling scheme and also for a given data set as follows:

$$
I_{1}(R)=V_{\text {posterior }(R)}\left(\ln T_{p}\right)
$$

here $R=\left(R_{1}, \ldots, R_{m}\right)$ denotes the sampling plan and $V_{\text {posterior }(R)}\left(\ln T_{p}\right)$ denotes the posterior variance of $\ln T_{p}$. Since the posterior variance of $\ln T_{p}$ depends on the data, we consider $E_{\text {data }}\left\{V_{\text {posterior }(R)}\left(\ln T_{p}\right)\right\}$, to make it a criterion, which is independent of data, see Kundu [9], i.e.

$$
C_{1}(R)=E_{\text {data }}\left\{V_{\text {posterior }(R)}\left(\ln T_{p}\right)\right\}
$$

One drawback about the Criterion 1 is that it is a function of the quantile point $p$. Using the idea of Gupta and Kundu [7] and Kundu [9], we propose Criterion 2, which is independent of $p$. 


\section{CRiterion 2:}

$$
C_{2}(R)=E_{\text {data }}\left\{\int_{0}^{1} V_{\text {posterior }(R)}\left(\ln T_{p}\right) d W(p)\right\} .
$$

In this case the weight function $0 \leq W(p) \leq 1$, is a non-negative weight function defined on on $[0,1]$ and it has to be decided before hand depending on the problem. For example if somebody is interested to give more stress at the center, then more stress should be given near $p=0.5$, on the other hand if somebody is interested to give more stress at the tails, then $W(\cdot)$ can be chosen accordingly.

Therefore, if we have two different censoring schemes, say $R^{(1)}$ and $R^{(2)}$, then $R^{(1)}$ is

better than $R^{(2)}$, with respect to the criterion 1, if $C_{1}\left(R^{(1)}\right)<C_{1}\left(R^{(2)}\right)$. Similarly, it is true for criterion 2. The main problem here is the computation of (17) and (18). We use Lindley's approximation and simulation technique to approximate (17) and (18) as given below.

\subsection{Finding the Optimum Scheme}

First we mention how to approximate (17) and (18) numerically. In the Appendix B, we have explained in details how to approximate $V_{\text {posterior }(R)}\left(\ln T_{p}\right)$ and $\int_{0}^{1} V_{\text {posterior }(R)}\left(\ln T_{p}\right) d W(p)$ using Lindley's approximation. The following Monte Carlo approximation method can be used to compute (17) and (18) numerically.

Monte Carlo Approximation:

- Step 1: Generate $\alpha$ and $\lambda$ from the joint prior distribution of $\alpha$ and $\lambda$ assumings that they are proper.

- Step 2: For the given censoring scheme, generate a progressively censored sample $t_{1}, \ldots, t_{m}$ when the lifetime distribution is GE with parameters $\alpha$ and $\lambda$. 
- Step 3: Calculate the approximate values of $V_{\text {posterior }(R)}\left(\ln T_{p}\right)$ and $\int_{0}^{1} V_{\text {posterior }(R)}\left(\ln T_{p}\right) d W(p)$ as suggested in Appendix B.

- Step 4: Repeat the procedure, say $N$ times and compute their averages.

Now to find the optimum censoring scheme with respect to Criterion 1 or Criterion 2, one needs to compute (17) or (18) for all possible censoring scheme and choose that one which has minimum (17) or (18). It should be noted that this is a discrete optimization problem and it can be solved in finite number of steps. Although the total number of sampling schemes are finite, they can be quite large. For fixed $m$ and $n$, total $\left(\begin{array}{c}n-1 \\ m-1\end{array}\right)$ possible progressive censoring schemes are available. For example, when $n=25$ and $m=12$, then the possible number of censoring schemes are $\left(\begin{array}{l}24 \\ 11\end{array}\right)=2496144$, which is quite large. Till date, We do not have any efficient algorithm to find the optimal censoring scheme in this case. We propose the following sub-optimal censoring scheme. Note that for fixed $n$ and $m$ all the censoring schemes of the form $\left(R_{1}, \ldots, R_{m}\right)$ such that $R_{1}+\ldots+R_{m}=n-m$, will belong to the convex hull generated by the points $(n-m, 0, \ldots, 0) \ldots(0, \ldots, 0, n-m)$. Therefore, a sub-optimal censoring scheme can be obtained by choosing the optimal censoring scheme among these extreme points on the convex hull.

For illustrative purposes, we have presented different values of $C_{1}(R)$ for different $p$ and $C_{2}(R)$ when $W(p)=1$ for all $0<p<1$. We have taken $n=20$ and $m=8$ and 12. The hyper parameters of $\alpha$ and $\lambda$ are $(a=1, b=2.5)$ and $(c=1, d=1)$ respectively. We have reported the results only for the extreme points in Table 5 and Table 6. It is clear that Type-III censoring provides the minimum variance (maximum information) in most of the cases and Type-II provides the maximum variance in all the cases among the sub-optimal schemes.

One natural question is, how to implement this optimum censoring procedure in practice, when the the priors are not known. In practice we can use independent gamma priors as 
Table 5: The values of $C_{1}(R)$ and $C_{2}(R)$ for different $R$, when $n=20$ and $m=8$.

\begin{tabular}{|c|c|c|c|c|c|c|}
\hline$R$ & \multicolumn{5}{|c|}{$C_{1}(R)$} & \multirow{2}{*}{$C_{2}(R)$} \\
\cline { 2 - 6 } & $p=0.50$ & $p=0.90$ & $p=0.95$ & $p=0.99$ & $p=0.999$ & \\
\hline$\left(12,7^{*} 0\right)$ & 0.3060 & 0.2918 & 0.2103 & 0.2001 & 0.1950 & 0.4616 \\
\hline$\left(0,12,6^{*} 0\right)$ & 0.3321 & 0.2338 & 0.2244 & 0.2147 & 0.2093 & 0.4651 \\
\hline$\left(0,0,12,5^{*} 0\right)$ & 0.3513 & 0.2502 & 0.2402 & 0.2300 & 0.2243 & 0.4845 \\
\hline$\left(3^{*} 0,12,4^{*} 0\right)$ & 0.3737 & 0.2663 & 0.2567 & 0.2453 & 0.2390 & 0.5100 \\
\hline$\left(4^{*} 0,12,3^{*} 0\right)$ & 0.3981 & 0.2830 & 0.2732 & 0.2606 & 0.2539 & 0.5394 \\
\hline$\left(5^{*} 0,12,0,0\right)$ & 0.4281 & 0.3054 & 0.2935 & 0.2793 & 0.2712 & 0.5771 \\
\hline$\left(6^{*} 0,12,0\right)$ & 0.4651 & 0.3307 & 0.3167 & 0.3022 & 0.2934 & 0.6235 \\
\hline$\left(7^{*} 0,12\right)$ & 0.5055 & 0.3568 & 0.3410 & 0.3253 & 0.3154 & 0.6754 \\
\hline
\end{tabular}

they are very flexible, but still we need to know the the corresponding hyper-parameters. If nothing is known, then some pilot survey can be carried out and using the integrated likelihood approach, estimates of the hyper-parameters can be obtained. Model misspecification is an important issue in this case, it has not been addressed here. More work is needed along that direction.

\section{Conclusions}

In this paper we have considered the Bayesian inference of the unknown parameters of the generalized exponential distribution when the data are progressively censored. Under the assumptions of independent gamma priors on both the shape and scale parameters the Bayes estimates are obtained using Lindley's approximation and also by importance sampling technique. It is observed that if the prior information are available, then the Bayes estimates have clear advantages over the MLEs.

We have also used two different criteria to choose the optimum censoring plans. It is observed that finding the optimum censoring scheme is a discrete optimization problem and we have suggested sub-optimal censoring scheme which can be obtained very easily. The 
Table 6: The values of $C_{1}(R)$ and $C_{2}(R)$ for $n=20$ and $m=12$.

\begin{tabular}{|c|c|c|c|c|c|c|}
\hline \multirow{2}{*}{$R$} & \multicolumn{5}{|c|}{$C_{1}(R)$} & \multirow{2}{*}{$C_{2}(R)$} \\
\cline { 2 - 6 } & $p=0.50$ & $p=0.90$ & $p=0.95$ & $p=0.99$ & $p=0.999$ & \\
\hline$\left(8,11^{*} 0\right)$ & 0.2128 & 0.1356 & 0.1286 & 0.1211 & 0.1169 & 0.3199 \\
\hline$\left(0,8,10^{*} 0\right)$ & 0.2111 & 0.1376 & 0.1311 & 0.1238 & 0.1201 & 0.3116 \\
\hline$\left(2^{*} 0,8,9^{*} 0\right)$ & 0.2133 & 0.1399 & 0.1333 & 0.1261 & 0.1226 & 0.3115 \\
\hline$\left(3^{*} 0,8,8^{*} 0\right)$ & 0.2176 & 0.1441 & 0.1369 & 0.1298 & 0.1257 & 0.3136 \\
\hline$\left(4^{*} 0,8,7^{*} 0\right)$ & 0.2221 & 0.1468 & 0.1401 & 0.1332 & 0.1289 & 0.3180 \\
\hline$\left(5^{*} 0,8,6^{*} 0\right)$ & 0.2242 & 0.1486 & 0.1410 & 0.1331 & 0.1289 & 0.3156 \\
\hline$\left(6^{*} 0,8,5^{*} 0\right)$ & 0.2321 & 0.1543 & 0.1471 & 0.1391 & 0.1346 & 0.3288 \\
\hline$\left(7^{*} 0,8,4^{*} 0\right)$ & 0.2375 & 0.1580 & 0.1503 & 0.1420 & 0.1377 & 0.3331 \\
\hline$\left(8^{*} 0,8,3^{*} 0\right)$ & 0.2438 & 0.1617 & 0.1540 & 0.1457 & 0.1413 & 0.3399 \\
\hline$\left(9^{*} 0,8,2^{*} 0\right)$ & 0.2498 & 0.1653 & 0.1572 & 0.1485 & 0.1443 & 0.3470 \\
\hline$\left(10^{*} 0,8,0\right)$ & 0.2557 & 0.1687 & 0.1603 & 0.1516 & 0.175 & 0.3538 \\
\hline$\left(11^{*} 0,8\right)$ & 0.2641 & 0.1738 & 0.1653 & 0.1564 & 0.1523 & 0.3641 \\
\hline
\end{tabular}

detailed comparison of the optimal and sub-optimal censoring schemes are in progress and it will be reported later.

\section{ACKNowledGements:}

The authors would like to thank two unknown referees and the guest editor for several helpful comments which had improved the earlier versions of the manuscript. Part of the work of the first author is supported by a grant from the Department of Science Technology, Government of India. 


\section{Appendix A}

For the two-parameter case, using the notation $\left(\lambda_{1}, \lambda_{2}\right)+(\alpha, \lambda)$, the Lindley's approximation can be written as

$$
\widehat{g}=g\left(\widehat{\lambda}_{1}, \widehat{\lambda}_{2}\right)+\frac{1}{2}\left(A+l_{30} B_{12}+l_{03} B_{21}+l_{21} C_{12}+l_{12} C_{21}\right)+p_{1} A_{12}+p_{2} A_{21},
$$

where

$$
\begin{gathered}
A=\sum_{i=1}^{2} \sum_{j=1}^{2} w_{i j} \tau_{i j}, \quad l_{i j}=\frac{\partial^{i+j} L\left(\lambda_{1}, \lambda_{2}\right)}{\partial \lambda_{i}^{i} \lambda_{2}^{j}}, i, j=0,1,2,3, \quad \text { and } \quad i+j=3, \quad p_{i}=\frac{\partial p}{\partial \lambda_{i}}, \quad w_{i}=\frac{\partial g}{\partial \lambda_{i}}, \\
w_{i j}=\frac{\partial^{2} g}{\partial \lambda_{i} \partial \lambda_{j}}, \quad p=\ln \pi\left(\lambda_{1}, \lambda_{2}\right), \quad A_{i j}=w_{i} \tau_{i i}+w_{j} \tau_{j i}, \quad B_{i j}=\left(w_{i} \tau_{i i}+w_{j} \tau_{i j}\right) \tau_{i i}, \\
c_{i j}=3 w_{i} \tau_{i i} \tau_{i j}+w_{j}\left(\tau_{i i} \tau_{j j}+2 \tau_{i j}^{2}\right) .
\end{gathered}
$$

In this case

$$
L(\alpha, \lambda)=m(\ln \alpha+\ln \lambda)-\lambda \sum_{i=1}^{m} t_{i}+\sum_{i=1}^{m}(\alpha-1) \ln \left(1-e^{-\lambda t_{i}}\right)+\sum_{i=1}^{m} R_{i} \ln \left(1-\left(1-e^{-\lambda t_{i}}\right)^{\alpha}\right) .
$$

Let us use the following notation for $i=1, \ldots, m$.

$$
\xi_{i}=1-e^{-\widehat{\lambda} t_{i}}, \quad \xi_{i}^{\prime}=t_{i} e^{-\widehat{\lambda} t_{i}}, \quad \xi_{i}^{\prime \prime}=-t_{i}^{2} e^{-\widehat{\lambda} t_{i}}, \quad \xi_{i}^{\prime \prime \prime}=t_{i}^{3} e^{-\widehat{\lambda} t_{i}}
$$

We have

$$
\tau_{11}=\frac{W}{U W-V^{2}}, \quad \tau_{12}=-\frac{V}{U W-V^{2}}, \quad \tau_{22}=\frac{U}{U W-V^{2}},
$$

where

$$
\begin{aligned}
U= & -\frac{\partial^{2} L}{\partial \alpha^{2}}=\frac{m}{\alpha^{2}}+\sum_{i=1}^{m} \frac{R_{i}\left(\ln \xi_{i}\right)^{2} \xi_{i}^{\alpha}}{\left(1-\xi_{i}^{\alpha}\right)^{2}} \\
V= & -\frac{\partial^{2} L}{\partial \alpha \partial \lambda}=-\sum_{i=1}^{m} \frac{\xi_{i}^{\prime}}{\xi_{i}}+\sum_{i=1}^{m} \frac{R_{i} \xi_{i}^{\prime} \xi_{i}^{\alpha-1}}{\left(1-\xi_{i}^{\alpha}\right)^{2}}\left[1-\xi_{i}^{\alpha}+\alpha \ln \xi_{i}\right] \\
W= & -\frac{\partial^{2} L}{\partial \lambda^{2}}=\frac{m}{\lambda^{2}}-(\alpha-1) \sum_{i=1}^{m} \frac{\xi_{i} \xi_{i}^{\prime \prime}-\left(\xi_{i}^{\prime}\right)^{2}}{\xi_{i}^{2}} \\
& +\alpha \sum_{i=1}^{m} \frac{R_{i} \xi_{i}^{\alpha-2}}{\left(1-\xi_{i}^{\alpha}\right)^{2}}\left[(\alpha-1)\left(\xi_{i}^{\prime}\right)^{2}+\xi_{i} \xi_{i}^{\prime \prime}-\xi_{i}^{\alpha+1} \xi_{i}^{\prime \prime}+\xi_{i}^{\alpha}\left(\xi_{i}^{\prime}\right)^{2}\right]
\end{aligned}
$$




$$
\begin{aligned}
l_{30}= & \frac{\partial^{3} L}{\partial \alpha^{3}}=\frac{2 m}{\alpha^{3}}-\sum_{i=1}^{m} \frac{R_{i}\left(\ln \xi_{i}\right)^{3} \xi_{i}^{\alpha}\left(1+\xi_{i}^{\alpha}\right)}{\left(1-\xi_{i}^{\alpha}\right)^{3}} . \\
l_{03}= & \frac{2 m}{\lambda^{3}}+(\alpha-1) \sum_{i=1}^{m} \frac{1}{\xi_{i}^{4}}\left(\left\{\xi_{i}^{\prime \prime \prime} \xi_{i}+\xi_{i}^{\prime \prime} \xi_{i}^{\prime}-2 \xi_{i}^{\prime} \xi_{i}^{\prime \prime}\right\} \xi_{i}^{2}-2 \xi_{i} \xi_{i}^{\prime}\left(\xi_{i} \xi_{i}^{\prime \prime}-\left(\xi_{i}^{\prime}\right)^{2}\right)\right) \\
& -\alpha \sum_{i=1}^{m} \frac{R_{i}}{\left(1-\xi_{i}^{\alpha}\right)^{3}}\left\{\left[(\alpha-1)(\alpha-2) \xi_{i}^{\alpha-3}\left(\xi_{i}^{\prime}\right)^{3}+3(\alpha-1) \xi_{i}^{\alpha-2} \xi_{i}^{\prime} \xi_{i}^{\prime \prime}+\xi_{i}^{\alpha-1} \xi_{i}^{\prime \prime \prime}\right.\right. \\
& \left.-(2 \alpha-1) \xi_{i}^{2 \alpha-2} \xi_{i}^{\prime} \xi_{i}^{\prime \prime}-\xi_{i}^{2 \alpha-1} \xi_{i}^{\prime \prime \prime}+(2 \alpha-2) \xi_{i}^{2 \alpha-3}\left(\xi_{i}^{\prime}\right)^{3}+2 \xi_{i}^{2 \alpha-2} \xi_{i}^{\prime} \xi_{i}^{\prime \prime}\right]\left(1-\xi_{i}^{\alpha}\right) \\
& \left.+2 \alpha \xi_{i}^{\prime} \xi_{i}^{\alpha-1}\left[(\alpha-1) \xi_{i}^{\alpha-2}\left(\xi_{i}^{\prime}\right)^{2}+\xi_{i}^{\alpha-1} \xi_{i}^{\prime \prime}-\xi_{i}^{2 \alpha-1} \xi_{i}^{\prime \prime}+\xi_{i}^{2 \alpha-2}\left(\xi_{i}^{\prime}\right)^{2}\right]\right\} . \\
l_{12}= & \sum_{i=1}^{m} \frac{1}{\xi_{i}^{2}}\left(\xi_{i}^{\prime \prime} \xi_{i}-\left(\xi_{i}^{\prime}\right)^{2}\right)-\sum_{i=1}^{m} \frac{R_{i}}{\left(1-\xi_{i}^{\alpha}\right)^{3}}\left[\left\{\left(\xi_{i}^{\alpha-1} \xi_{i}^{\prime \prime}+\left(\xi_{i}^{\prime}\right)^{2}(\alpha-1) \xi_{i}^{\alpha-2}\right)\left(1-\xi_{i}^{\alpha}+\alpha \ln \xi_{i}\right)\right.\right. \\
+ & \left.\left.\alpha\left(\xi_{i}^{\prime}\right)^{2} \xi_{i}^{\alpha-2}\left(1-\xi_{i}^{\alpha}\right)\right\}\left(1-\xi_{i}^{\alpha}\right)+2 \alpha\left(\xi_{i}^{\prime}\right)^{2} \xi_{i}^{2 \alpha-2}\left(1-\xi_{i}^{\alpha}+\alpha \ln \xi_{i}\right)\right] . \\
l_{21}= & -\sum_{i=1}^{m} \frac{R_{i} \xi_{i}^{\prime}}{\left(1-\xi_{i}^{\alpha}\right)^{3}}\left[2 \xi_{i}^{\alpha-1} \ln \xi_{i}\left(1-\xi_{i}^{\alpha}\right)+\alpha \xi_{i}^{\alpha-1}\left(\ln \xi_{i}\right)^{2}\left(1+\xi_{i}^{\alpha}\right)\right] .
\end{aligned}
$$

Now when $g(\alpha, \lambda)=\alpha$, we have

$$
w_{1}=1, w_{2}=0, w_{i j}=0, i, j=1,2
$$

therefore,

$$
A=0, B_{12}=\tau_{11}^{2}, B_{21}=\tau_{21} \tau_{22}, C_{12}=3 \tau_{11} \tau_{12}, C_{21}=\left(\tau_{22} \tau_{11}+2 \tau_{21}^{2}\right) A_{12}=\tau_{11}, A_{21}=\tau_{12} .
$$

Now (8) follows by using

$$
p_{1}=\left(\frac{b-1}{\widehat{\alpha}}-a\right) \quad \text { and } \quad p_{2}=\left(\frac{d-1}{\widehat{\lambda}}-c\right) .
$$

For $(9)$, note that $g(\alpha, \lambda)=\lambda$; then

$$
w_{1}=0, w_{2}=1, w_{i j}=0, i, j=1,2
$$

and

$$
A=0, B_{12}=\tau_{12} \tau_{11}, B_{21}=\tau_{22}^{2}, C_{12}=\tau_{11} \tau_{22}+2 \tau_{12}^{2}, C_{21}=3 \tau_{22} \tau_{21} A_{12}=\tau_{21}, A_{21}=\tau_{22} .
$$




\section{Appendix B}

In this Appendix first we provide the approximation of $V_{\text {posterior }(R)}\left(\ln T_{p}\right)$ using Lindley's technique and then along the same line the approximation of $\int_{0}^{1} V_{\text {posterior }(R)}\left(\ln T_{p}\right) d W(p)$ also can be obtained. Since

$$
V_{\text {posterior }(R)}\left(\ln T_{p}\right)=E_{\text {posterior }(R)}\left(\ln T_{p}\right)^{2}-\left(E_{\text {posterior }(R)}\left(\ln T_{p}\right)\right)^{2}
$$

we approximate $E_{\text {posterior }(R)}\left(\ln T_{p}\right)$ and $E_{\text {posterior }(R)}\left(\ln T_{p}\right)^{2}$ separately. We will use (19) in both the cases. For that purpose we just need to specify $w_{1}, w_{2}, w_{11}, w_{12}, w_{21}, w_{22}$ only. The rest of the quantities are already available in Appendix A.

Approximating $E_{\text {posterior }(R)}\left(\ln T_{p}\right)$ :

$$
g(\alpha, \lambda)=T_{p}=\ln \left(-\ln \left(1-p^{\frac{1}{\alpha}}\right)\right)-\ln \lambda=\ln (-\ln u(\alpha))-\ln \lambda=v(\alpha)-\ln \lambda,
$$

where

$$
u(\alpha)=1-p^{\frac{1}{\alpha}}, \quad v(\alpha)=\ln (-\ln u(\alpha)) .
$$

Note that

$$
u^{\prime}(\alpha)=\frac{1}{\alpha^{2}} p^{\frac{1}{\alpha}}(\ln p), \quad u^{\prime \prime}(\alpha)=-\frac{1}{\alpha^{4}} \times\left[p^{\frac{1}{\alpha}}(\ln p)^{2}+2 \alpha p^{\frac{1}{\alpha}}(\ln p)\right]
$$

Therefore, in this case

$$
\begin{gathered}
w_{1}=\frac{u^{\prime}(\alpha)}{\ln u(\alpha) u(\alpha)}=a_{1}(\alpha) \quad(\text { say }), \quad w_{2}=-\frac{1}{\lambda}, \\
w_{11}=\frac{u^{\prime \prime}(\alpha) u(\alpha) \ln u(\alpha)-u^{\prime}(\alpha)\left(u^{\prime}(\alpha) \ln u(\alpha)+u^{\prime}(\alpha)\right)}{(u(\alpha)(\ln u(\alpha)))^{2}}=b_{1}(\alpha) \quad \text { (say) } \\
w_{22}=\frac{1}{\lambda^{2}}, \quad w_{12}=w_{21}=0 .
\end{gathered}
$$

Approximating $E_{\text {posterior }(R)}\left(\ln T_{p}\right)^{2}$ :

$$
g(\alpha, \lambda)=(v(\alpha)-\ln \lambda)^{2} .
$$


In this case

$$
\begin{gathered}
w_{1}=2 a_{1}(\alpha)(v(\alpha)-\ln \lambda), \quad w_{2}=\frac{2}{\lambda}(\ln \lambda-v(\alpha)), \\
w_{11}=2\left(a_{1}(\alpha)^{2}+b_{1}(\alpha) v(\alpha)\right)-2 b_{1}(\alpha) \ln \lambda, \quad w_{12}=-\frac{2}{\lambda} a_{1}(\alpha)=w_{21}, \quad w_{22}=\frac{2}{\lambda^{2}}(1-\ln \lambda+v(\alpha)) .
\end{gathered}
$$

Now to approximate $\int_{0}^{1} V_{\text {posterior }(R)}\left(\ln T_{p}\right) d W(p)$, note that

$$
\int_{0}^{1} V_{\text {posterior }(R)}\left(\ln T_{p}\right) d W(p)=\int_{0}^{1} E_{\text {posterior }(R)}\left(\ln T_{p}\right)^{2} d W(p)-\int_{0}^{1}\left(E_{\text {posterior }(R)}\left(\ln T_{p}\right)\right)^{2} d W(p) .
$$

Therefore $\int_{0}^{1} V_{\text {posterior }(R)}\left(\ln T_{p}\right) d W(p)$ can be approximated using numerical integration with respect to the weight function $W(\cdot)$.

\section{References}

[1] Balakrishnan, N. (2007), "Progressive Censoring Methodology: An Appraisal", (with discussions), Test, vol. 16, no. 2, 211 - 296.

[2] Balakrishnan, N. and Aggrawala, R. (2000), Progressive Censoring, Theory, Methods and Applications, Birkhauser, Boston.

[3] Balakrishnan, N. and Sandhu, R.A. (1995), "A simple algorithm for generating progressively type-II generated samples", American Statistician, vol. 49, 229 - 230.

[4] Balasooriya, U. and Balakrishnan, N. (2000), "Reliability sampling plan for log-normal distribution", IEEE Transactions on Reliability, vol. 49, 199 - 203.

[5] Berger, J.O., and Sun, D. (1993), "Bayesian Analysis for the PolyWeibull Distribution", Journal of the American Statistical Association, vol. 88, 14121418.

[6] Gupta, R.D. and Kundu, D. (1999), "Generalized exponential distribution", Australian New Zealand Journal of Statistics, vol. 41, 173 - 188. 
[7] Gupta, R.D. and Kundu, D. (2006), "Comparison of the Fisher information matrices of the Weibull and GE distributions", Journal of Statistical Planning and Inference, vol. 136 , no. $9,3130-3144$.

[8] Gupta, R.D. and Kundu, D. (2007), "Generalized exponential distribution; existing methods and some recent developments", Journal of Statistical Planning and Inference, vol. 137, no. $11,3537-3547$.

[9] Kundu, D. (2008), "Bayesian inference \& life testing plan for Weibull distribution in presence of progressive censoring", Technometrics, vol. 50, 144-154.

[10] Kundu, D. and Pradhan, B. (2008), "Estimating the parameters of the generalized exponential distribution in presence of hybrid censoring", Communications in Statistics - Theory and Methods (to appear).

[11] Lawless, J.F. (2003), Statistical models and methods for lifetime data, 2-nd edition, John Wiley and Sons, New York.

[12] Madi, M. T. and Raqab, M. Z. (2007), "Bayesian Prediction of Rainfall Records Using the Generalized Exponential Distribution", Environmetrics, vol .18, 541-549.

[13] Mitra, S. and Kundu, D. (2008), "Analysis of the left censored data from the generalized exponential distribution", Journal of Statistical Computation and Simulation, vol. 78, no. $7,669-679$.

[14] Ng, T., Chan, C.S. and Balakrishnan, N. (2004), "Optimal progressive censoring plans for the Weibull distribution", Technometrics, vol. 46, 470 - 481.

[15] Pradhan, B. and Kundu, D., "On progressively censored generalized exponential distribution", TEST, DOI:10.1007/s11749-008-0110-1. 
[16] Son, Y.S. and Oh, M. (2006), "Bayes estimation of the two-parameter gamma distribution", Communications in Statistics - Theory and Methods, vol. 35, 285 - 293.

[17] Zhang, Y. and Meeker, W.Q. (2005), "Bayesian life test planning for the Weibull distribution with given shape parameter", Metrika, vol. 61, 237 - 249. 